\title{
Tetracycline-tooth interaction: An elemental analysis from prenatal period to early childhood
}

\author{
Kiyoshi Mochizuki, Hiromichi Fujii, Yasuyo Mizuguchi, Yukio Machida and Masashi Yakushiji \\ Department of Pediatric Dentistry, Tokyo Dental College \\ 1-2-2 Masago, Mihama-ku, Chiba 261-8502, JAPAN
}

\begin{abstract}
This study was performed to observe the dental hard tissue of newborn rats after their mothers were administered demethylchlortetracycline (DTC) during pregnancy. Undecalcified ground sections were observed by microradiography, and tetracycline-stained tooth was determined by ultraviolet light. The mineralization pattern and the element distribution in the enamel and dentin of the colored tooth were analyzed using an electron probe X-ray microanalyzer (XMA). Decalcified serial sections were stained with hematoxylin and eosin (HE). The results obtained showed that tetracycline caused abnormalities in the enamel and dentin formation. The longer period of tetracycline administration was tended to be associated with more pronounced coloration, and this was associated with the moderate alteration of calcification in some areas of the enamel and dentin, as observed on contact microradiograms (CMR). The Mg levels were higher, while those of $\mathrm{P}$ were lower for the DTC experimental groups than the control group. However, the Ca levels were comparable between the experimental and the control groups.
\end{abstract}

\author{
Key words \\ Medication administered prenatally \\ and postnatally, \\ Rat, \\ Tetracycline, \\ $\mathrm{X}$-ray microanalyzer
}

\section{Introduction}

The effectiveness of the oral administration of tetracycline for treating fungal or larval infections has been documented. The usefulness of the local administration of tetracycline in the oral cavity for the treatment of periodontal disease caused by Rickettsia rickettsii, R.japonica, and $R$. tsutsugamushi has been reported. Tetracycline administration in children during odontogenesis may lead to coloration and odontogenesis imperfecta in deciduous and permanent teeth ${ }^{1-5)}$. The relationship between drug administration and odontogenic abnormalities has not been elucidated thus far ${ }^{6-13)}$. Few studies have investigated the effects of maternal administration of tetracycline on the formation of tooth enamel and dentin in the fetus ${ }^{14)}$. Therefore, this study analyzed tooth enamel and dentin formation using

Received on September 28, 2005

Accepted on January 12, 2006 an electron probe X-ray microanalyzer (XMA) to determine the effects of tetracycline administration on odontogenesis both pre- and postnatally in case if the mother is administered tetracycline during pregnancy and only postnatally if the neonates are administered tetracycline.

\section{Materials and Methods}

Demethylchlortetracycline (DTC) powder was provided by Wyeth Lederle (Japan Co., Ltd.); it was dissolved in sterile purified water and placed in a bottle. Wistar Fischer rats (F344/DuCrj, Charles River Japan Co., Ltd.) were used in this study. These rats were divided into 4 groups. Each group comprised 10 newborns rats and included of pregnant female.

Group I: DTC was continuously administered to the pregnant female Fischer rats at the beginning of the fetal age of day 14. It is thought that odontogenesis begins until delivery. 




Fig. 1 Medication term of tetracycline

Table 1 Medication quantitative of tetracycline

\begin{tabular}{cccc}
\hline Medication term & $\begin{array}{c}\text { Prenatal period at } \\
14 \text { days-Birth }\end{array}$ & Birth-3 weeks & 3 weeks-5 weeks \\
\hline Group I & $\begin{array}{c}247.5 \mathrm{mg} \\
(35.36 \mathrm{mg} / 200 \mathrm{~g} \cdot \text { day })\end{array}$ & - & - \\
\hline Group II & $\begin{array}{c}233.0 \mathrm{mg} \\
(33.23 \mathrm{mg} / 200 \mathrm{~g} \cdot \text { day })\end{array}$ & $\begin{array}{c}1,468.5 \mathrm{mg} \\
(69.93 \mathrm{mg} / 200 \mathrm{~g} \cdot \text { day })\end{array}$ & - \\
\hline Group III & - & $1,182.5 \mathrm{mg}$ & $1,347.5 \mathrm{mg}$ \\
& - & $(56.30 \mathrm{mg} / 200 \mathrm{~g} \cdot$ day $)$ & $(13.75 \mathrm{mg} / 45 \mathrm{~g} \cdot$ day $)$ \\
\hline
\end{tabular}

Prenatal period at 14 days-Birth: Oral administration to the mother's body Birth-3 weeks, 3 weeks-5 weeks: Oral administration to a newborn baby

Group II: DTC was continuously administered to the pregnant female Fischer rats from the beginning of the odontogenesis until weaning.

Group III: DTC was continuously administered to the female Fischer rats from delivery until weaning and then to their newborns from weaning until the age of 5 weeks.

Group IV (control group): The pregnant females and their newborns were administered sterile purified water.

Drinking water containing DTC was prepared by dissolving DTC in sterile purified water. The drinking water was changed on a daily basis throughout the study, and the total dose of orally administered DTC was calculated by measuring the amount of drinking water consumed daily. The administration term is shown in Figure 1, and the total DTC dose is shown in Table 1. In all 4 groups, the newborns were weaned for 3 weeks after birth and then raised and observed macroscopically until the age of 5 weeks. At 5 weeks, the rats were sacrificed using a lethal dose of ether, fixed in $10 \%$ neutral buffered formalin, and then dehydrated. The left teeth were decalcified and embedded in paraffin for serial sectioning to obtain $5 \mu \mathrm{m}$ thick slices; these sections were stained with hematoxylin and eosin (HE). The right teeth were dehydrated, embedded in Polyester resin (Rigolac 2004 and Rigolac 70F; Okenshoji Co., Ltd.), and $40 \mu \mathrm{m}$ faciolingual sections were prepared on a Leitz 1600 saw microtome for undecalcified sections. The undecalcified ground sections were observed by microradiography, and tetracycline labeling was evaluated microscopically under ultraviolet light. Simultaneously, contact microradiograms (CMR) were obtained using a Softex CMR (Nihon Softex Co., Ltd.) under the following conditions: focus/film distance, $2 \mathrm{~cm}$; tube voltage, $5 \mathrm{kV}$; tube current, 


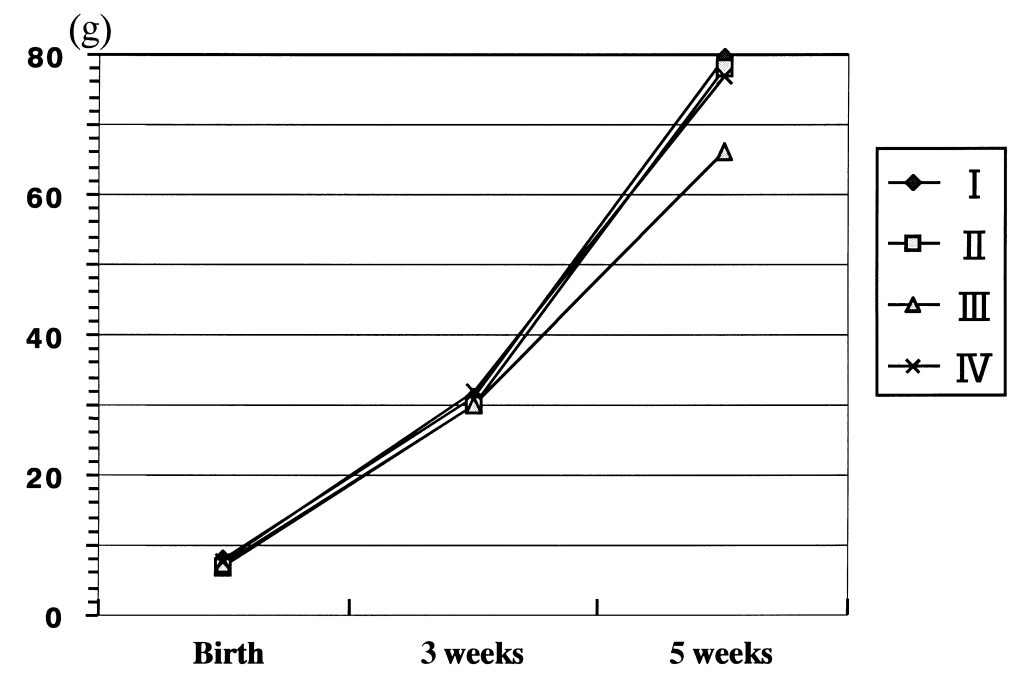

Fig. 2 Changes in the body weight of the newborn rats

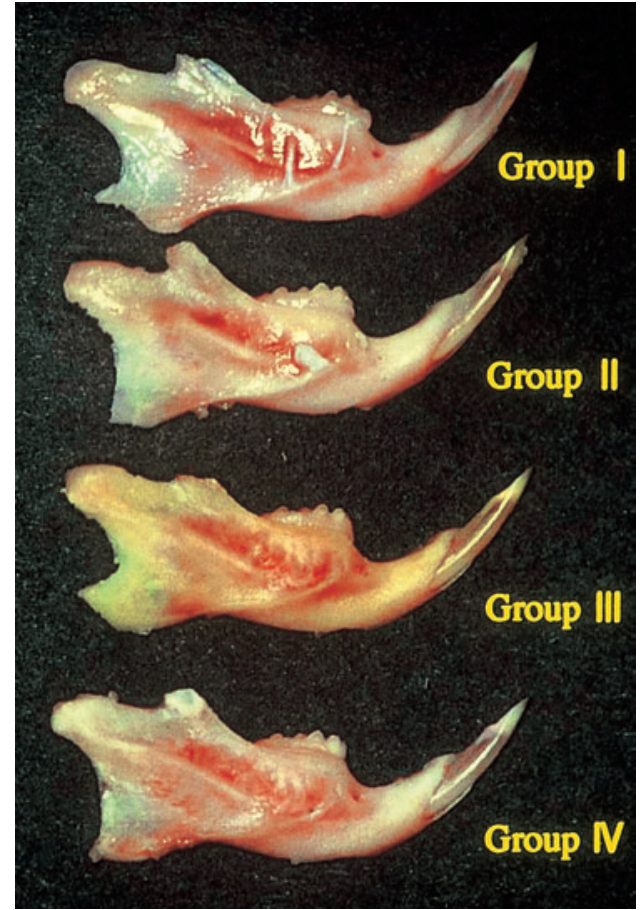

Fig. 3 Macroscopic examination

The longer period of tetracycline administration was tended to be associated with more pronounced coloration.

$5 \mathrm{~mA}$; and irradiation time, $10 \mathrm{~min}$. High-resolution CMR films (SO-343, Kodak) were subjected to specific image processing and then mounted using Canada balsam. CMR films were analyzed under a light microscope, and the undecalcified specimens were examined under a fluorescence microscope.
Subsequently, the undecalcified specimens were subjected to vacuum deposition using carbon, and an elemental analysis was carried out using an XMA (X-3010, Hitachi Co., Ltd.). The document feed speed and chart speed for the XMA were set at $150 \mu \mathrm{m} / \mathrm{min}$ and $5 \mathrm{~cm} / \mathrm{min}$, respectively. The images of the line analyses of $\mathrm{Ca}, \mathrm{P}$, and $\mathrm{Mg}$ were scanned using a Scanner (GS-2200, Epson Co., Ltd.) attached to a PC (Power Book G3, Macintosh). Subsequently, 3 types of charts were displayed on 1 screen using graphic software (FreeHand 7, Macromedia Co., Ltd.).

After 3 weeks, the rats were provided with solid feed (MF; Oriental Kobo). The animals were handled in accordance with the animal experimentation guidelines established by the Ministry of Health and Welfare of Japan.

\section{Results}

Figure 2 shows the changes in the body weight of the newborns, and Figure 3 shows the coloration of teeth at the end of the observation period and the macroscopic findings. Although the average body weight of the newborns in Group III (the group with the highest total DTC dose) was the lowest, there were no significant differences in the body weight among the 4 experimental groups. Although neither neonatal anomaly nor loss of dental parenchyma were evident, the group receiving the highest total DTC dose tended to show pronounced tooth coloration. The degree of coloration was strongest 


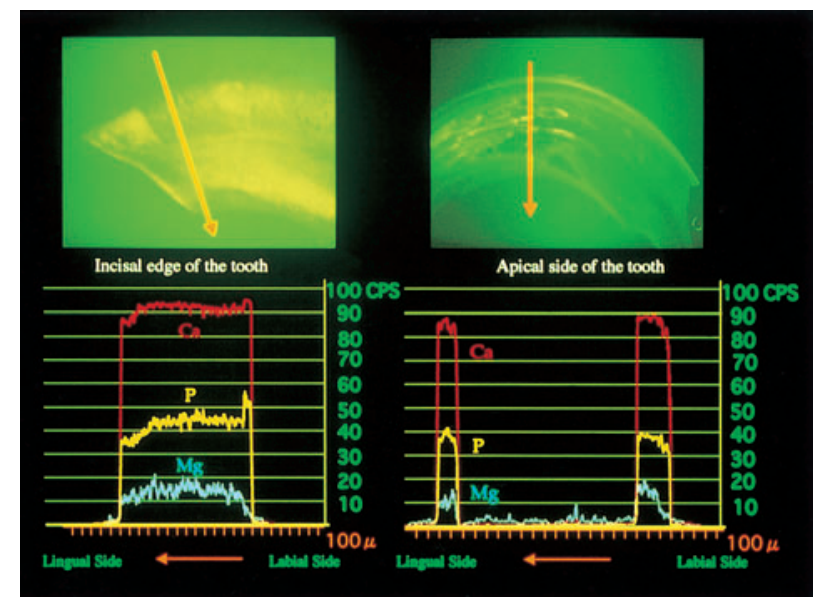

Fig. 4 XMA analyses of the Group I

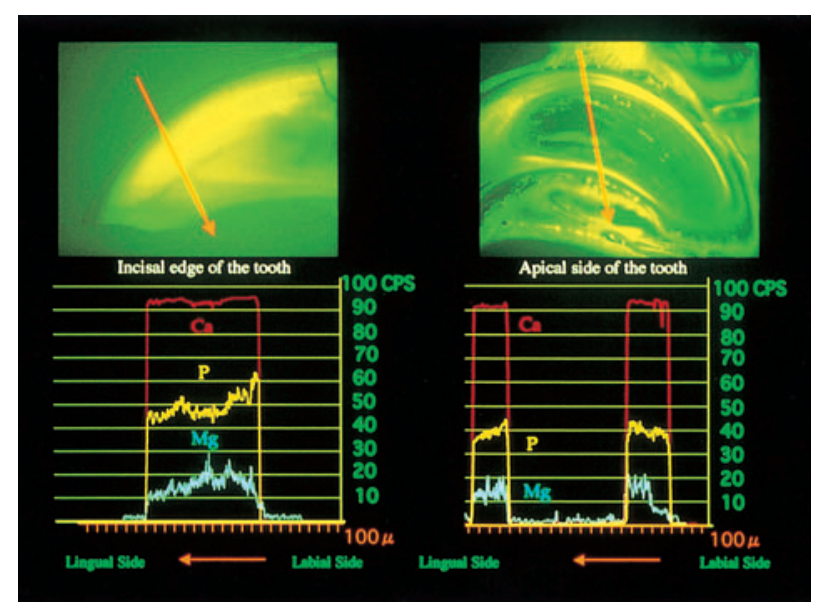

Fig. 6 XMA analyses of the Group III

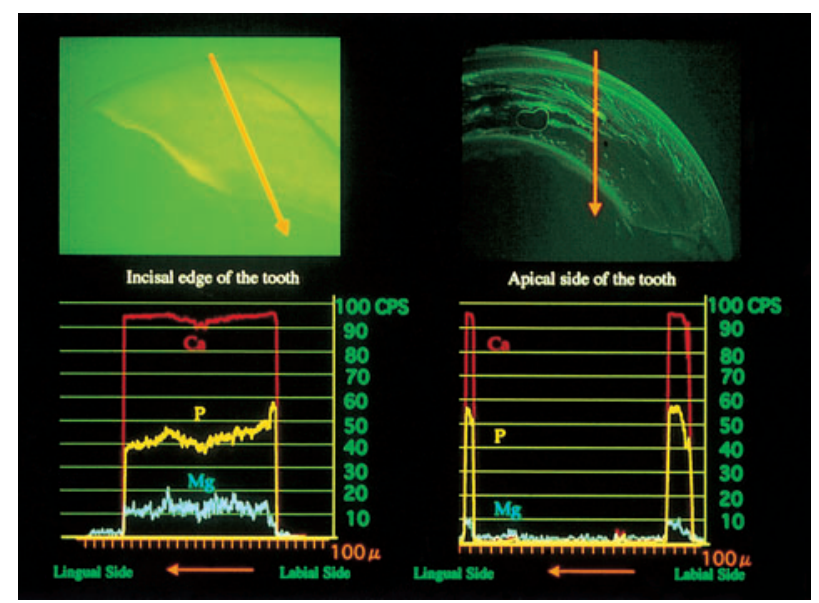

Fig. 8 XMA analyses of the Group IV

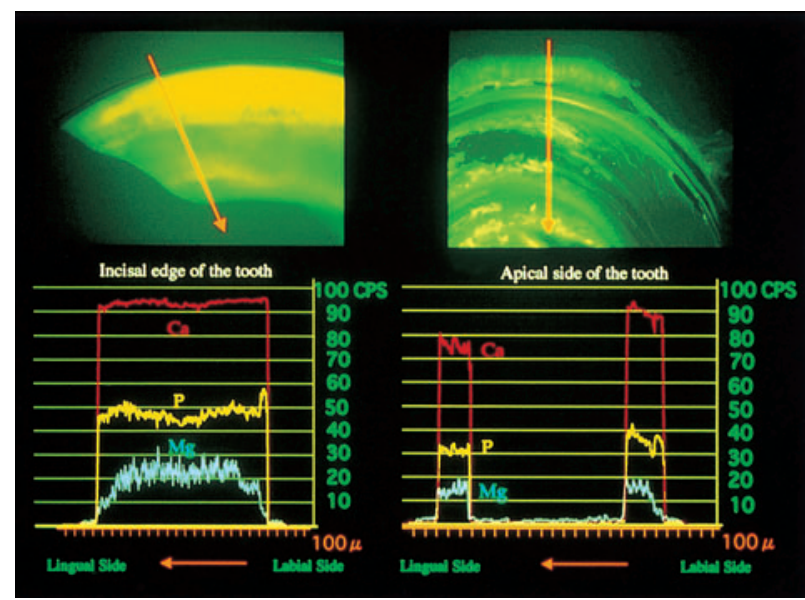

Fig. 5 XMA analyses of the Group II

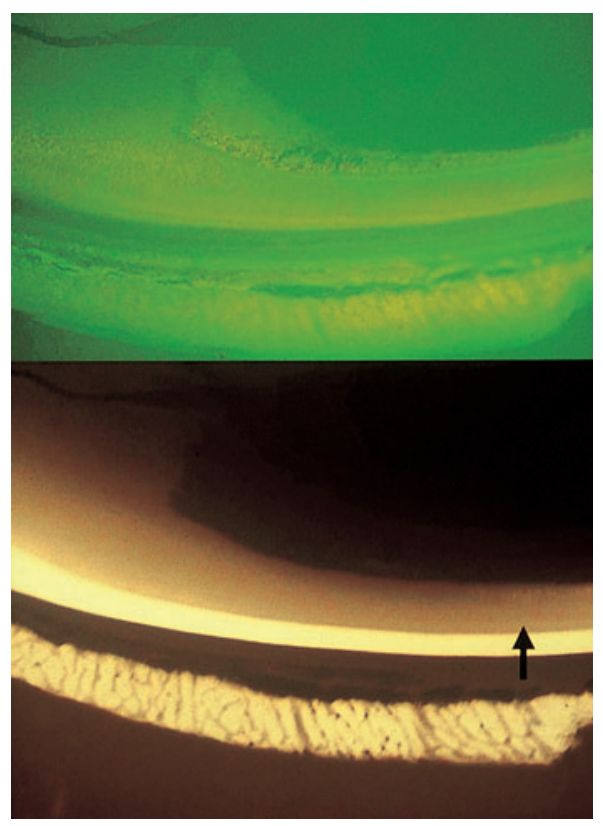

Fig. 7 Tetracycline labeling and microradiography, Group III (moderate alteration of calcification: arrow)

for Group III followed by Group II and then Group I.

The results of fluorescence and line analyses performed using an electron probe XMA are described as follows. The DTC labeling for Group I is shown in Figure 4. DTC was continuously administered to pregnant rats from the beginning of odontogenesis until delivery. In Group I, a mild DTC labeling was observed in the enamel on the side of the incisal edge, and a diffused and wavy labeling was observed in the one-third of the enamel and the dentin on the side of the root. Line analyses 




Fig. 9 Hematoxylin and eosin stain, incisal side

were performed between the labial and lingual sides using the line drawn on a fluorescent scan. On the side of the incisal edge of the teeth, the $\mathrm{Ca}$ and $\mathrm{P}$ levels in the enamel were elevated, and these levels decreased with the gradient from the labial dentin to the dental pulp. However, the $\mathrm{Ca}$ and $\mathrm{P}$ levels were low in the immature enamel wherein calcification occurs on the side of the root, while these levels were high in the dentin on the side of the root. The $\mathrm{Mg}$ level was low in the enamel on the side of the incisal edge and the root, and changes in the $\mathrm{Mg}$ level in the dentin when compared to the controls were similar to those observed for P.

Figure 5 shows the results obtained for Group II, in which DTC was continuously administered to the mother rats from the beginning of odontogenesis until weaning. From the dentin on the side of the incisal edge to the dentin on the side of the root, the layered labeling lines were observed along with a highly diffuse fluorescent image. As compared to Group I, the wavy and diffuse labeling of the enamel in Group II was broader and more fluorescent. The results of a line analysis showed that changes in the $\mathrm{Ca}, \mathrm{P}$, and $\mathrm{Mg}$ levels on the side of the incisal edge and the root when compared to the controls were similar to the changes in Group I.

Figure 6 shows the results obtained for Group III, in which DTC was continuously administered to female rats after the delivery until weaning and to the newborns from weaning to the age of 5 weeks. The coloration and fluorescence of the teeth were most pronounced in this group. The highly diffuse fluorescent and wavy labeling, which was more pronounced than in Group II, was observed in all

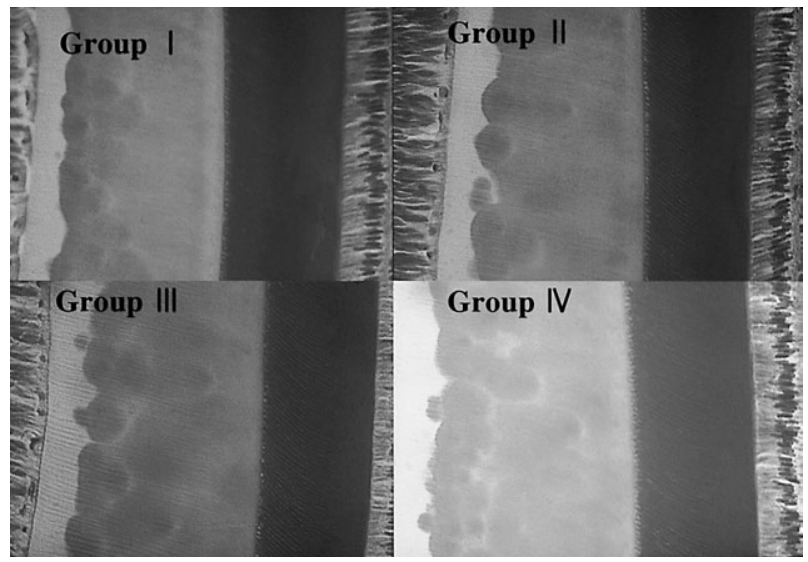

Fig. 10 Hematoxylin and eosin stain, apical side

dental layers, with the exception of the enamel on the side of the incisal edge. The layered labeling was observed on the half of the enamel on the root side. The CMR observations (Figure 7) showed changes in the calcification of the dentin on the side of the root edge (arrow) when compared with the controls; the degree of change was greater than that observed in Groups I and II. However, calcification was not apparent in the areas with the layered labeling lines in the enamel. The results of a line analysis showed that changes in the $\mathrm{Ca}$ and $\mathrm{P}$ levels on the incisal edge and side of the root were similar to those in Groups I and II. In addition, the Mg level was higher in the areas having high fluorescence dentin on the side of the incisal edge.

Figure 8 shows the results obtained for Group IV, in which DTC was not administered. The changes in the $\mathrm{Ca}$ and $\mathrm{P}$ levels in the dentin on the side of the incisal edge were almost similar between Groups III and IV; however, the Mg level in the dentin on the side of the incisal edge in Group III was higher than that in Group IV. Moreover, the Mg level in the dentin on the side of the root in Group III was higher than that in Group IV.

The results of XMA-assisted line analyses were compared in the 4 groups (I, II, III, and IV). There were no significant differences in the $\mathrm{Ca}$ and $\mathrm{P}$ levels in the experimental groups. However, the $\mathrm{Mg}$ level in the areas of dentin with DTC labeling was high, while the P level in these areas of dentin was low, and this tendency was marked on the side of the root in the experimental groups. In Group III, in which DTC was administered to female rats from after delivery until weaning and then to their newborns 
from weaning to the age of 5 weeks, the $\mathrm{Mg}$ level in dentin with DTC deposition was twice as that in Group IV (control group). In addition, the $\mathrm{Mg}$ level in dentin with DTC deposition was slightly higher in Group I (DTC administered to pregnant rats prenatally) than that in Group IV.

The results of HE staining showed an alternation of hematoxylin-stained layers with eosin-stained layers (striped pattern) in some areas of irregular dentin formation wherein the incisal edge pulp was plugged. In particular, this tendency was apparent in Group III, in which DTC was administered to the mother rats from after the delivery to weaning and to their newborns from weaning to the age of 5 weeks. The changes in the dentin staining, in comparison with the controls, were low in Group II and Group I. The HE staining of the one-third of the enamel on the side of the root and the surrounding dentin in the experimental groups (Groups I, II, and III) was comparable to that of the control group (Group IV); i.e., there were no abnormal findings in this area (Figures 9 and 10).

\section{Discussion}

The formation of the dental enamel and dentin has been experimentally shown by an X-ray permeation band, indicating an area of low calcification, on the CMR of undecalcified specimens. In this study, changes in the calcified area of enamel and dentin were assessed using CMR, and the changes in dentin were evaluated using HE staining. Furthermore, the analysis of elements in enamel and dentin was performed using an XMA.

Tetracycline is chemically unique because it is a fluorescent material that can form a chelate bond with bivalent positive ions such as $\mathrm{Ca}^{++}$or $\mathrm{Mg}^{++}$. The antibacterial activity of tetracycline is mostly attributable to its ability to inhibit the synthesis of proteins by forming chelate bonds with positive ions such as $\mathrm{Ca}^{++}$or $\mathrm{Mg}^{++}$in bacteria. This prevents the binding of the aminoacyl tRNA with the mRNA ribosome complex, thus, preventing the translation of mRNA ${ }^{15,16)}$. With regard to the dental problems associated with the use of tetracycline, when tetracycline is absorbed during calcification of dentine, enamel, cementum, or bone, these tissues acquire a yellow color $^{2-13)}$. The degree of coloration is strongest in case of chlortetracycline administration, followed by tetracycline, demethylchlortetracycline, and oxytetracycline, in that order ${ }^{17}$. This study was conducted using demethylchlortetracycline.

There have been contradictory reports on the effects of tetracycline on teeth. In one study, when tetracycline was administered during the formation of hard enamel and dentin, the teeth became less resistant to dental caries and their hardness reduced $^{5}$. Magnesium in dentin exists mostly on the surface of apatite crystals and was considered to catalyze alkali phosphatase ${ }^{18-20)}$. Therefore, $\mathrm{Mg}$ was intimately involved in the calcification of teeth, and it has been reported that the Mg level was low in areas with elevated $\mathrm{Ca}$ and $\mathrm{P}$ levels. However, no significant differences were observed in the $\mathrm{Ca}$ levels among these 4 groups. The $\mathrm{Mg}$ level in the areas of dentin with DTC labeling was higher, while the P level in the areas of dentin was lower in the administration groups than those observed in the control group in this study.

The degree of calcification abnormality as observed by Löfgren et al. . $^{\text {) }}$ with intravenous administration of DTC was not observed in the present results, possibly due to the oral administration of DTC. Marked calcification abnormalities were not seen, although DTC was administered for a long period at time and its total dose was high. When tetracycline was administered orally during fetal odontogenesis, coloration abnormalities and the presence of tetracycline were detected by fluorescence analysis; however, the incidence of calcification abnormalities was low. There was a tendency for longer period of tetracycline administration to be associated with more pronounced coloration and for more pronounced coloration to be associated with moderate alteration of calcification in some areas of enamel and dentin, as seen on CMR. Suga ${ }^{21)}$ reported that the tetracycline that was deposited during the enamel formation in tooth was removed from the enamel matrix when the organic materials in the enamel were removed during adulthood. The degree of change in the enamel observed in this study was therefore less than expected, given the results obtained by Suga's ${ }^{19-21)}$ study and the fact that DTC was administered orally in this study.

The results showed an alternation of hematoxylinstained layers and eosin-stained layers that correlated with the circadian rhythm ${ }^{22,23)}$ in some areas of irregular dentin formation in which the pulp toward the incisal edge was packed. The growth pattern of dentin is believed to be closely correlated with the cyclic changes in the secretion of hormones or other 
substances. Ohtsuka ${ }^{23)}$ concluded that the body's biological clock affects dentin formation and acts directly or indirectly via the endocrine system to produce the circadian cyclic structure of dentin. In this study, distinct alternation of light and dark layers was observed for Group III. On the other hand, the degree of staining changes in the dentin on the side of the incisal edge, as compared to the controls, was low for Groups II and I.

In this study, marked calcification abnormalities accompanying parenchymal loss were not seen, although DTC was administered for a long time and when its total dose was high. Alternating hematoxylin-stained layers and eosin-stained layers were observed in areas of demineralized irregular dentine in which the pulp toward the incisal edge was packed; these layers were rapidly formed by attrition. These findings showed that tetracycline caused abnormalities accompanied by staining changes when absorbed by the rapidly forming dental enamel and dentin.

In addition, when DTC was orally administered, the element composition of the affected teeth was different from that of the normally calcified teeth.

\section{References}

1) Shwachman, H. and Schuster, A.: The tetracyclines; applied pharmacology. Pediatr Clin North Am 3: 295-303, 1956.

2) Weyman, J. and Porteous, J.R.: Discoloration of teeth possibly due to administration of tetracyclines; a preliminary report. Brit Dent J 113: 51-54, 1962.

3) Harcourt, J.K. and Johnson, N.W.: In vivo incorporation of tetracycline in the teeth of man. Arch Oral Biol 7: 431-437, 1962.

4) Gregg, J.M. and Avery, J.K.: Studies of alveolar bone growth and tooth eruption using tetracyclineinduced fluorescence. J Oral Ther Pharmacol 1: 264, 1964.

5) Baker, K.L.: The fluorescent, microradiographic, microhardness and specific gravity properties of tetracycline-affected human enamel and dentine. Arch Oral Biol 17: 525-536, 1972.

6) Owen, L.N.: The effects of administering tetracyclines to young dogs with particular preference to localization of the drugs in the teeth. Arch Oral Biol 8: 715-727, 1963.

7) Bennett, I.C. and Law, D.B.: Incorporation of tetracycline in developing dog enamel and dentin.
J Dent Res 44: 788-793, 1965.

8) Hammarström, L.: Tetracycline in developing rat enamel in relation to protein synthesis and maturation. Acta Odont Scand 26: 337-356, 1968.

9) Löfgren, C.G., Omnell, K.A. and Nylen, M.U.: Effect of intraperitoneal injections of tetracycline hydrochloride and oxytetracycline on forming enamel of rat incisors. Calcif Tissue Res 2: 145-156, 1968.

10) Nylen, M.U., Omnell, K.A. and Löfgren, C.G.: An electron microscopic study of tetracycline-induced enamel defects in rat incisor enamel. Scand J Dent 80: 384-409, 1972.

11) Kruger, B.J.: Dose-dependent ultrastructural changes induced by tetracycline in developing dental tissues of the rat. J Dent Res 54: 822-828, 1975.

12) Westergaard, J.: Structural changes induced by tetracycline in secretory ameloblasts in young rats. Scand J Dent Res 88: 481-495, 1980.

13) Kallenbach, E.: Microscopy of tetracycline-induced lesions in the rat incisor enamel organ. Arch Oral Biol 24: 869-876, 1980.

14) Bevelander, G.: The effect of tetracycline on mineralization and growth. Adv Oral Biol 1: 205223, 1964.

15) Avery, J.K.: Oral Development and Histology. Thieme Medical Publishers, New York, 1994, pp. 135-138.

16) Tsutsumi, S., Itoh, H., Maehashi, H., Nakai, Y. and Matsumoto, Y.: Textbook of Dental Pharmacology. Gakken Shoin, Tokyo, 1981, pp.356-358. (in Japanese)

17) Weyman, J.: The clinical appearance of tetracycline staining of the teeth. Brit Dent J 118: 289-291, 1965.

18) Johansen, E.: Ultrastructural and chemical observations on dental caries. In: Mechanisms of Hard Tissue Destruction. A.A.A.S., Washington, 1963, pp. 182-211.

19) Suga, S., Soejima, H. and Tanaka, Y.: Electron probe $\mathrm{X}$-ray microanalysis of developing, erupted and carious dental hard tissues. J Dent Res 46: 12511252, 1967.

20) Suga, S., Kondo, M., Onodera, A., Kubota, Y. and Ohtsuka, M.: Electron microprobe analyses on the distributions of $\mathrm{Cl}, \mathrm{Mg}$ and $\mathrm{Na}$, in the enamels of various animals. Jpn J Oral Biol 13: 85-94, 1971. (in Japanese)

21) Suga, S. and Murayama, Y.: Microradiographical labeling study on the mineralization of guinea pigs enamel. Odontology 53: 154-162, 1965.

22) Shinoda, H. and Ogura, H.: A study on the circadian rhythms of dentine formation in rodents. Jpn J Oral Biol 19: 481-489, 1977. (in Japanese)

23) Ohtsuka, M. and Shinoda, H.: Ontogeny of circadian dentinogenesis in the rat incisor. Arch Oral Biol 40: 481-485, 1995. 